\title{
Estudiantes con discapacidad en una universidad chilena: desafíos de la inclusión ${ }^{1}$
}

\author{
Students with disabilities in a Chilean university \\ Gabriela Paulina VILLAFAÑE HORMAZÁBAL, Angélica Aurora CORRALES \\ HUENUL y Valentina Javiera SOTO HERNÁNDEZ \\ Universidad de Concepción (Chile)
}

Recibido: Septiembre 2014

Evaluado: Diciembre 2014

Aceptado: Diciembre 2014

\begin{abstract}
Resumen
El propósito de este artículo es dar cuenta de una investigación acerca de las condiciones de inclusión para los estudiantes con discapacidad en una universidad chilena. La investigación tiene un diseño cuantitativo, descriptivo y transversal. Para la recolección de datos se elaboró una encuesta que fue aplicada a 38 estudiantes con discapacidad. Los principales resultados revelan un alto nivel de retención de los estudiantes, quienes presentan una percepción positiva de su inclusión en la vida universitaria, así como una alta satisfacción con la mayoría de los servicios. Se destaca que siete de cada diez encuestados reconocen haber recibido algún tipo de atención educativa para realizar sus estudios. Sin embargo, existe una falta de articulación entre las iniciativas de la Universidad para favorecer el acceso y permanencia de personas con discapacidad; a esto se agrega la carencia de protocolos y de instancias de capacitación para profesores y administrativos. Se propone que la institución establezca un sistema de gestión que defina objetivos, estrategias y acciones para contribuir a la inclusión de personas con discapacidad.
\end{abstract}

Palabras clave: inclusión, estudiantes, discapacidad, educación superior, atención educativa.

\begin{abstract}
The purpose of this article is to give a report about a research related with the conditions of inclusion of students with disability in a Chilean university. This research is a quantitative, descriptive and cross-sectional study. To collect the data required, a survey was developed, which was applied to 38 students with disability. The main results reveal a high retention rate of students, who exhibit a positive perception of their inclusion in their university life and also a high level of satisfaction with most of the services provided. Seven out of ten students surveyed recognize having received some sort of education care from their programs to pursue their studies. However, there still exists a lack of connection between the current initiatives
\end{abstract}

\footnotetext{
${ }^{1}$ Este artículo se enmarca en el proyecto de investigación "Diagnóstico del estado actual de estudiantes con Necesidades Educativas Especiales asociadas Discapacidad", financiado por el Convenio de Desempeño "Profesores UdeC: Protagonistas del cambio en la sociedad del conocimiento".
} 
developed at the university to support the enrollment and permanence of students. Added to this fact, there is a lack of protocols and training for teachers and staff. In this study it is proposed that the university must establish a management system that defines objectives, strategies and actions that contribute to improve inclusion of people with disabilities.

Keywords: inclusion, students, disabilities, higher education, education care.

La inclusión de personas con discapacidad en el sistema escolar chileno ha sido impulsada desde las políticas públicas hace más de dos décadas, atendiendo a los compromisos asumidos por el estado. Sin embargo, los programas para promover la inclusión han avanzado lentamente en el logro de sus objetivos. A nivel de educación superior, las pocas cifras disponibles revelan que la inclusión es un asunto de desarrollo incipiente en las universidades. A la falta de estadísticas se suma una insuficiente producción científica que permita conocer las condiciones físicas, psicológicas, sociales y económicas de este grupo. En este contexto, se realizó un estudio sobre estudiantes con discapacidad en una universidad chilena, de manera de aportar información pertinente y actualizada, que permita realizar un primer diagnóstico de las condiciones de inclusión.

La investigación fue desarrollada a partir de la pregunta: ¿Cuáles son las condiciones de inclusión para los estudiantes con discapacidad en la universidad estudiada? Pregunta que implica otras como: ¿Qué tipo de discapacidad presentan los estudiantes? ¿Cuáles son los mecanismos facilitadores existentes en la institución? ¿Cuál es la percepción de los estudiantes respecto a las condiciones de inclusión que ofrece la universidad? Dadas estas premisas, el objetivo general de la investigación fue determinar las condiciones de inclusión para los estudiantes con discapacidad en la universidad estudiada. Los objetivos específicos fueron los siguientes: (1) Identificar y caracterizar a los estudiantes con discapacidad. (2) Identificar y analizar la atención educativa que ofrece la universidad a los estudiantes con discapacidad. (3) Determinar la satisfacción de los estudiantes con discapacidad respecto a la atención educativa y servicios otorgados (4) Establecer la percepción de los estudiantes con discapacidad acerca de su inclusión.

El artículo se organiza del siguiente modo: primero, se presenta un marco referencial; enseguida, , se abordan las políticas públicas desarrolladas en favor de la inclusión, y los resultados de las investigaciones más recientes sobre educación superior y discapacidad. Segundo, se presenta la metodología de investigación, procedimiento e instrumento utilizado. Tercero, se detallan los principales resultados. En el apartado de discusión y conclusiones, se analizan las condiciones actuales y los desafíos institucionales para la inclusión de personas con discapacidad.

\section{Marco referencial}

Las convenciones internacionales y normativas nacionales reconocen el derecho de las personas con discapacidad a la educación y a la no-discriminación. La labor de la Organización de las Naciones Unidades ha sido central, como lo manifiesta el Programa de Acción Mundial para las Personas con Discapacidad (1983) y las Normas 
Uniformes sobre la Igualdad de Oportunidades para las Personas con Discapacidad (1993).

La Conferencia Mundial sobre Necesidades Educativas Especiales (NEE) realizada en Salamanca (1994), promueve que las NEE asociadas y no asociadas a discapacidad sean consideradas como elementos relevantes de las políticas educativas de los países participantes. La Conferencia se planificó para dar respuesta a dos preocupaciones: (1) Garantizar a todos los niños, en particular a los niños con NEE, el acceso a las oportunidades de educación. (2) Promover educación de calidad.

La Convención sobre los Derechos de las Personas con Discapacidad del año 2006, señala que "los Estados Partes reconocen el derecho de las personas con discapacidad a la educación. Con miras a hacer efectivo este derecho sin discriminación y sobre la base de la igualdad de oportunidades, los Estados Partes asegurarán un sistema de educación inclusivo a todos los niveles así como la enseñanza a lo largo de la vida” (Art. 24).

Ainscow y Echeita (2010) plantean que mientras en la Declaración de Salamanca la inclusión educativa fue concebida como un principio, es decir, un criterio que orienta las políticas y estrategias educativas, la Convención de los Derechos de las Personas con Discapacidad (ONU, 2006) establece la educación inclusiva como un derecho positivo. Esto implica la obligación para los estados de crear las condiciones efectivas que garanticen este derecho.

La Conferencia Internacional de Educación (2008) y Ainscow y Milles (2008), plantean cuatro elementos que permiten comprender de mejor forma el concepto de educación inclusiva, en atención a su polisemia:

1. La inclusión es un proceso, por lo que puede concebirse como una búsqueda de las mejores formas de atender la diversidad del estudiantado.

2. La inclusión implica la búsqueda de la presencia, participación y éxito de todos los estudiantes.

3. La inclusión precisa de identificación y eliminación de barreras. El concepto de barreras es clave en tanto son estas barreras las que impiden el ejercicio del derecho a la educación inclusiva.

4. La inclusión pone particular énfasis en aquellos grupos de alumnos que podrían estar en riesgo de marginalización, exclusión o fracaso escolar.

Para Echeita (2013) la educación inclusiva es una perspectiva que aboga por superar la exclusión educativa; y tiene como foco los sujetos y colectivos que han sido privados del derecho a la educación (Escudero y Martínez, 2011). La inclusión se constituye en un "proceso para aprender a vivir con las diferencias de las personas (...) por tanto, de humanización y supone respeto, participación y convivencia” (López, 2011, p. 41).

Miles y Singal (2010) sostienen que la educación inclusiva es una perspectiva que promueve los principios democráticos, así como la igualdad y justicia social, de modo que todos los estudiantes puedan participar del proceso de enseñanza y aprendizaje. Para Unesco: 
"La educación inclusiva es esencial a la instauración de una educación de alta calidad para todos los estudiantes y al desarrollo de sociedades más inclusivas. Es crucial para alcanzar la equidad social y hace parte integral del aprendizaje a lo largo de la vida” (Unesco, 2009, p. 5).

Un rasgo esencial de la educación inclusiva, de acuerdo a Casanova (2011), es el cambio en relación al enfoque de la integración: el énfasis pasa desde el alumno hacia la institución. En otras palabras: "implica necesariamente (...) poner en marcha y sostener procesos cíclicos de innovación, mejora y reformas educativas (Echeita, et al., 2013, p. 350).

De acuerdo a Echeita et al. (2013), a pesar de los esfuerzos para que la educación inclusiva sea un enfoque vinculado al reconocimiento y valoración de la diversidad, los variados significados del concepto dan cuenta que este objetivo es lejano. Como resultado se produce una "dispersión de esfuerzos (teóricos, investigadores y prácticos)” (p. 104).

\section{Políticas públicas para la inclusión de estudiantes con discapacidad}

El Estado chileno ratificó los compromisos asumidos en las convenciones ya mencionadas, a través de la promulgación de la Ley sobre Igualdad de Oportunidades e Inclusión Social de Personas con Discapacidad, del año 2010, que sustituyó a la Ley de Integración Social de Personas con Discapacidad. Esta normativa asumió como objetivo "asegurar el derecho a la igualdad de oportunidades de las personas con discapacidad, con el fin de obtener su plena inclusión social, asegurando el disfrute de sus derechos y eliminando cualquier forma de discriminación fundada en la discapacidad”. Esta ley fomenta la "adopción de medidas de acción positiva orientadas a compensar las desventajas de una persona con discapacidad” (ONU, 2008).

A nivel de sistema escolar, la ley indica que "en su conjunto, deberá brindar alternativas educacionales a aquellos educandos que presenten necesidades educativas especiales" (Art.1). En particular, "las instituciones de educación superior deberán contar con mecanismos que faciliten el acceso de las personas con discapacidad, así como adaptar los materiales de estudio y medios de enseñanza para que dichas personas puedan cursar las diferentes carreras” (Art. 39).

Desde el punto de vista del derecho a la educación de las personas con discapacidad, un avance relevante fue la adopción de la Política Nacional de Educación Especial del Ministerio de Educación (MINEDUC), cuyo objetivo general es "hacer efectivo el derecho a la educación, a la igualdad de oportunidades, a la participación y a la no discriminación de las personas que presentan Necesidades Educativas Especiales, garantizando su pleno acceso, integración y progreso en el sistema educativo" (MINEDUC, 2005). Este plan materializó la implementación de mecanismos que aseguren la igualdad de oportunidades de educación para todos. La adopción de esta política implicó el progresivo abandono del "enfoque médico o rehabilitador, para ubicarse más bien en un marco predominantemente educativo" (Comisión de Expertos de Educación Especial, 2004). 
De acuerdo a FONADIS (2004), este derecho está lejos de ser cumplido: en Chile sólo el 8,5\% de las personas con discapacidad está estudiando actualmente, a diferencia del $27,5 \%$ de la población total del país. De este modo, por cada persona con discapacidad que estudia, cuatro no lo hacen. Adicionalmente, una de cada dos personas con discapacidad ha completado la Enseñanza Básica, una de cada ocho ha cursado Enseñanza Media completa y sólo una de cada veinte personas con discapacidad ha logrado acceder a la Educación Superior.

Recientemente, la Política Nacional para la Inclusión Social de las Personas con Discapacidad (2013-2020) definió que "el limitado acceso a la educación, particularmente en el nivel inicial y en la educación superior, sumado a la necesidad de mejorar las respuestas educativas que reciben los estudiantes con discapacidad en la modalidad de educación especial, son factores que intervienen decisivamente en los procesos de exclusión social que viven las personas con discapacidad (p. 27). En este contexto, se establecieron los siguientes lineamientos:

a) Incluir a las personas con discapacidad en el sistema general de educación, en todos los niveles y modalidades, velando por la permanencia y egreso del sistema.

b) Proveer servicios, recursos humanos, técnicos, conocimientos especializados y ayudas necesarias para fomentar, de acuerdo a la normativa vigente, un aprendizaje de calidad para niños, niñas y jóvenes con NEE asociadas a un déficit o a una discapacidad.

Para enfrentar el bajo ingreso a la educación superior de las personas con discapacidad, el Servicio Nacional de Discapacidad ${ }^{2}$ (2013) ha implementado acciones tales como la creación de una Red de Educación Inclusiva, la realización de Encuentros regionales de educación superior inclusiva, y un plan de continuidad para estudiantes con discapacidad en educación superior.

\section{Inclusión de estudiantes con discapacidad en la Educación Superior chilena}

El Informe sobre la Educación Superior en América Latina y el Caribe 2000-2005, indica que investigaciones de diversos países latinoamericanos revelan una baja incorporación de personas con discapacidad en la educación superior. A esto se agrega la falta de registros que proporcionen datos estadísticos confiables y detallados sobre la población, no sólo de estudiantes, sino de profesores y funcionarios con discapacidad (Moreno, 2005).

En Chile, se realizó un primer estudio nacional sobre discapacidad (FONADIS, 2004), que dedicó un apartado a la educación superior. Entre los principales resultados se destaca que un $92 \%$ de personas con discapacidad no estudia. En efecto, los estudiantes con discapacidad tienen 2 años menos de escolaridad que las personas sin discapacidad. Sólo un 7\% de las personas en esta condición declaró haber accedido a la educación superior, lo cual es explicado por dos factores: la alta deserción escolar durante la enseñanza básica, y la edad de las personas con discapacidad (la mayoría

\footnotetext{
${ }^{2}$ En adelante, SENADIS y anteriormente llamado Fondo Nacional de Discapacidad (FONADIS).
} 
supera los 30 años). Del total de estudiantes que accedieron a la educación superior, dos de cada tres ingresa a la universidad. . Sin embargo, un $60 \%$ de ellos no completó sus estudios. La discapacidad más frecuente se relaciona con problemas de visión (67\%), seguida de limitaciones de desplazamiento (39\%), problemas de audición (20\%), relaciones con otros (18\%), cuidado personal (14\%), comprensión y comunicación (14\%). Además, la condición socio-económica es identificada como una variable que afecta significativamente el acceso a la educación superior de este grupo.

La investigación realizada por Barros (2011) sobre inclusión de estudiantes con discapacidad en instituciones de educación superior, revela que la matrícula total asciende a 843 , de los cuales el $52 \%$ es de sexo masculino y el $48 \%$ de sexo femenino. Los tipos de discapacidad más frecuentes son la motora (41\%) y la visual (28\%).

Las investigaciones recientes en torno a la inclusión de personas con discapacidad en la educación superior, revelan las dificultades para implementar las herramientas que favorezcan la inclusión, pero asimismo, demuestran la voluntad de los distintos actores de propiciar la participación de las personas con discapacidad.

Ocampo (2012) analizó las percepciones de estudiantes y profesores respecto a la inclusión de personas con discapacidad, concluyendo que 9 de cada 10 estudiantes están completamente de acuerdo con el deber de la universidad de facilitar el acceso, y ofrecer oportunidades de inclusión. Respecto a los docentes y directivos, el autor plantea que presentan una actitud de apertura frente a las posibilidades de acceso de estudiantes con discapacidad, pero que no cuentan con los mecanismos de intervención para generar planes estratégicos de inclusión.

Tapia y Manosalva (2012) realizaron un estudio en dos universidades chilenas no especificadas. Los resultados plantean que los estudiantes con discapacidad perciben dificultades en el sistema de selección universitaria debido a la falta de una legislación que lo regule. No obstante, destacan el compromiso de los profesores y compañeros de curso durante el proceso formativo. Respecto a las estrategias implementadas en las universidades estudiadas, una de ellas se encuentra en un "proceso de integración de personas con y sin discapacidad”, a través de acciones como la sensibilización a la comunidad. La segunda universidad presenta una trayectoria de 10 años en materia de integración, por lo que sus acciones se concentran en aspectos pedagógicos y prestación de servicios. Según los autores, las debilidades en la implementación de las políticas de integración se vinculan a aspectos como infraestructura, didáctica y adecuación de instrumentos de evaluación.

La investigación realizada por Salazar y otros (2013), analizó la percepción de un grupo de estudiantes con discapacidad respecto de las prácticas de inclusión de la Universidad Austral de Chile. Los estudiantes perciben que existen múltiples barreras que limitan su formación, principalmente de acceso y currículo, quienes presentan bajo nivel de satisfacción respecto a las políticas institucionales de integración puesto que no ofrecen atención educativa adecuada a sus necesidades.

Salinas y otros (2013) realizaron una investigación cualitativa a estudiantes con discapacidad en dos universidades chilenas. Los entrevistados concibieron el ingreso a la educación superior como el logro de una meta, y como el desafío de una nueva 
experiencia. Para comprender el proceso de inclusión se propuso una aproximación a través del modelo ecológico, a partir del cual se identificaron factores externos, definidos como facilitadores y obstaculizadores. Entre los facilitadores, además de la familia y los pares, destaca la existencia de programas de apoyo a estudiantes con discapacidad. Respecto a los obstaculizadores, existe escasa formación a los profesores en estrategias educativas para estudiantes con discapacidad.

\section{Metodología}

La investigación se realizó de acuerdo a una metodología cuantitativa y corresponde a un estudio descriptivo, los cuales "miden o evalúan diversos aspectos, dimensiones o componentes del fenómeno o fenómenos a investigar" (Hernández y otros, 1991, p. 60). Para Vieytes (2004, p.93), los estudios descriptivos buscan "un conocimiento exhaustivo del objetivo o alguno de sus atributos".

La principal técnica de recolección de datos utilizada fue una encuesta de tipo descriptiva, que, en correspondencia con el tipo de estudio definido, tiene como propósito “describir con precisión un fenómeno" (Vieytes, 2004, p. 329).

A fin de obtener una visión acabada de los procesos de inclusión, se decidió encuestar a la totalidad de alumnos con discapacidad de la universidad, por lo que el universo fueron todos los estudiantes en esta condición. Respecto al desarrollo temporal, la encuesta es de tipo transversal, puesto que la medición se realizó en un tiempo único.

Un estudiante con discapacidad fue entendido como aquel que requiere atención educativa referida a adaptaciones de acceso al currículum y/o adecuación e implementación a la infraestructura.

\section{Procedimientos}

En la primera parte de la investigación se diseñó y aplicó una encuesta a los 93 jefes de carrera de la institución, cuya finalidad fue identificar a los estudiantes con discapacidad. La universidad no posee un registro de la condición de discapacidad de sus estudiantes, lo que implicó este paso previo. De este modo, y mediante una encuesta realizada online, se pudo identificar a estos estudiantes, y posteriormente fueron invitados a participar en la investigación. Luego se diseñó una encuesta para realizar la recolección de datos.

Los estudiantes fueron contactados mediante el siguiente protocolo. Primero, vía email institucional, presentando la investigación y la importancia de su participación. Segundo, solicitando la colaboración del jefe de carrera, quien convocaba directamente al estudiante para ser encuestado. Tercero, mediante una llamada telefónica a los estudiantes, invitándolos a participar. La encuesta fue aplicada a 38 estudiantes que participaron voluntariamente de los 40 identificados, en condición de alumnos regulares del año académico 2013. La encuesta se realizó cara a cara, en un mismo 
lugar que fue previamente definido y estuvo a cargo de un grupo de tesistas de último año de la carrera de Educación Diferencial de la institución.

Respecto a los resguardos éticos de la investigación, cabe destacar que los estudiantes encuestados mostraron su acuerdo con las condiciones del estudio mediante la lectura y posterior firma del Consentimiento Informado, que declara que su participación en la encuesta es voluntaria. Vinculado a lo anterior, la investigación fue aprobada por el Comité de Ética de la institución.

\section{Instrumento}

El equipo de investigación diseñó una encuesta para aplicar a los estudiantes con discapacidad identificados en la primera etapa. La primera parte corresponde a preguntas de tipo socio-demográfico y de trayectoria escolar. La segunda parte indaga en el nivel de satisfacción de los estudiantes respecto a 19 elementos de infraestructura de los campus, destinados a personas con discapacidad; luego acerca de los servicios prestados por la universidad. Enseguida, se consulta por la participación en actividades extra programáticas ofrecidas por la universidad. En una cuarta parte, se consulta sobre las percepciones del proceso de inclusión en la universidad.

La encuesta fue sometida a validación de juicio de expertos, mediante un proceso que contó con representantes del MINEDUC, SENADIS, profesionales y académicos de la universidad estudiada, así como un profesional con discapacidad. Dos tareas fueron asignadas a los jueces expertos. La primera, realizada individualmente, consistió en determinar la pertinencia y claridad de los ítems incluidos en el instrumento. Estos aspectos fueron determinados por los jueces de acuerdo a los objetivos específicos. Las opciones de respuesta se constituyeron a partir de una escala de cero a dos; donde cero indicaba que la cualidad era inexistente; uno, que manifestaba la cualidad, pero no a cabalidad; y dos, cuando la cualidad se expresaba adecuadamente.

La segunda tarea consistió en presentar las respuestas de los jueces, las que alcanzaron un alto consenso en los aspectos analizados. No obstante, los jueces recomendaron incluir nuevos ítems en otras secciones, los que fueron aprobados. En una segunda ronda de consultas, se alcanzó consenso respecto a la versión final de la encuesta.

Los resultados presentados en este artículo responden a los objetivos específicos ya señalados, por lo que dan cuenta de una parte de la investigación total.

\section{Principales resultados}

\section{Caracterización de los estudiantes encuestados}

La encuesta aplicada revela que el mayor número de estudiantes con discapacidad se encuentra en el Campus Uno (28 estudiantes), seguido del Campus Tres (9 estudiantes) y finalmente, el Campus Dos (1 estudiante), lo que representa un 74\%, $24 \%$ y 3\%, respectivamente. El Campus Uno y Tres, concentran estudiantes con distintos tipos de discapacidad, mientras que el Campus Dos sólo presenta un 
estudiante con diversidad funcional por movilidad reducida. Respecto a las unidades que tienen mayor número de estudiantes con discapacidad, destacan la Facultad de Educación, Ciencias Jurídicas y Administrativas, y Escuela de Educación.

\begin{tabular}{clcc}
\hline Campus & \multicolumn{1}{c}{ Facultad } & Frecuencia & Porcentaje \\
\hline \multirow{4}{*}{ Uno } & Educación & 8 & 21,1 \\
& Ciencias Física y Matemáticas & 4 & 10,5 \\
& Ciencias Económicas y Administrativas & 1 & 2,6 \\
& Humanidades y Arte & 3 & 7,9 \\
& Ciencias Químicas & 1 & 2,6 \\
& Ciencias Jurídicas y Sociales & 7 & 18,4 \\
& Agronomía & 1 & 2,6 \\
& Ingeniería & 1 & 2,6 \\
& Arquitectura, Urbanismo y Geografía & 1 & 2,6 \\
& Ciencias Sociales & 1 & 2,6 \\
\hline \multirow{2}{*}{ Tres } & Escuela de Educación & 6 & 15,8 \\
& Escuela de Ciencias y Tecnología & 3 & 7,9 \\
\hline Dos & Escuela de Administración y Negocios & 1 & 2,6 \\
\hline \multirow{2}{*}{ Total } & $\mathbf{3 8}$ & $\mathbf{1 0 0}$
\end{tabular}

Tabla 1: Facultades/Escuelas de procedencia de los estudiantes encuestados Fuente: Encuesta a estudiantes con discapacidad de la institución, año académico 2013.

En relación al año de ingreso, se destaca que un 37\% comenzó sus estudios durante los años 2012 y 2013. Por tanto, son estudiantes que cursan la primera mitad de su carrera. Por otro lado, un 31\% de los encuestados ingresó entre 2005 y 2008, por lo que han permanecido a lo menos seis años en la institución. De hecho, los más antiguos ingresaron el año 2005, por lo que han permanecido nueve años en la universidad. Aquellos que ingresaron durante 2010 y 2011, representan un 22\% de los encuestados.

Respecto al sexo de los encuestados, un 68\% corresponde a hombres y $32 \%$ a mujeres. Este dato revela una diferencia notoria respecto al porcentaje nacional de mujeres con algún tipo de discapacidad en el sistema de Educación Superior en Chile, donde se ha constatado una participación casi igualitaria en términos de género. En efecto, el año 2009, el número de mujeres matriculadas en la educación superior superó al número de hombres, tendencia que se mantiene actualmente (CNED, 2014).

En cuanto a la edad de los encuestados, un 13\% tiene 19 años o menos; 50\% presenta entre 20 y 24 años; un 29\% tiene entre 25 y 29 años; y un 5\% tiene entre 30 y 34 años. Finalmente, un 2.6\% presenta más de 40 años.

En relación al tipo de residencia de los estudiantes, un $47 \%$ vive con sus padres, un $18 \%$ vive con otros familiares, un $18 \%$ en hogar universitario, un $11 \%$ habita en 
pensión y un 5\% arrienda departamento /casa compartida sin familiares. Por tanto, casi 3 de cada 4 estudiantes viven con familiares.

Respecto al tipo de discapacidad, un $40 \%$ de los encuestados presenta discapacidad visual, seguido de un $32 \%$ con diversidad funcional por movilidad reducida (discapacidad física) y un 18\%, discapacidad psíquica, principalmente Síndrome de Asperger. Existe un 8\% que presentan otros tipos de discapacidad. Finalmente, un 3\%, es decir, solo un estudiante, presenta discapacidad auditiva (Gráfico 1). Un 95\% de los encuestados ha sido diagnosticado por un médico, y un $63 \%$ del total se encuentra inscrito en el Registro Nacional de la Discapacidad. ${ }^{3}$

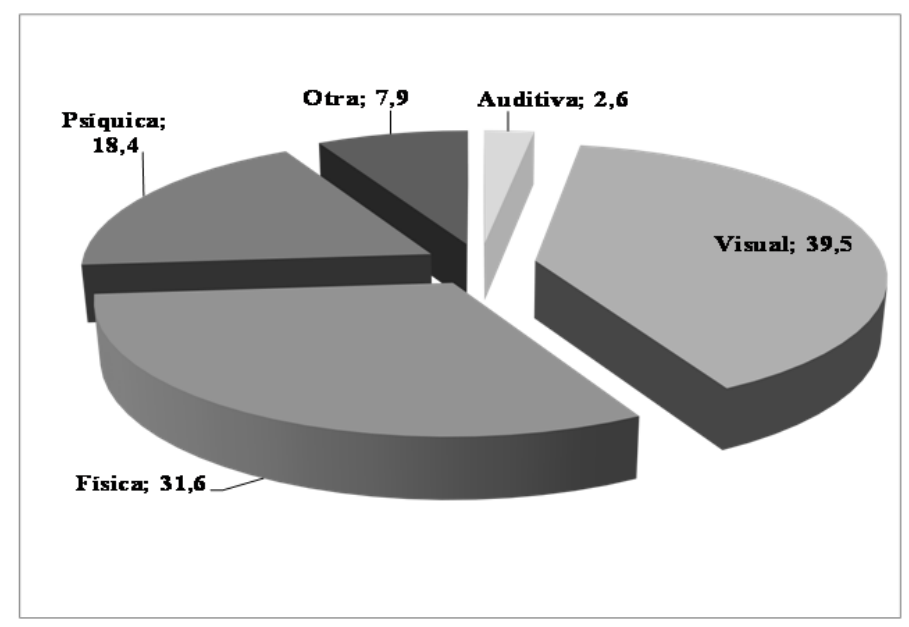

Gráfico 1: Tipo de discapacidad de estudiantes encuestados

Fuente: Encuesta a estudiantes con discapacidad de la institución, año académico 2013.

En cuanto a la trayectoria escolar de los estudiantes encuestados, se destaca que un 42\% asistió a Programas de Integración Escolar ${ }^{4}$. En otras palabras, del total de encuestados, un 26\% participó en planes de integración durante Enseñanza Básica y Media, seguido de un 11\% que participó solamente en Enseñanza Media.

Sobre el establecimiento educacional de procedencia, se destaca que un $42 \%$ egresó de la enseñanza media desde un establecimiento escolar municipal y un 53\% de un

\footnotetext{
${ }^{3}$ Este Registro es administrado por el Registro Civil, y mediante la incorporación de antecedentes de personas naturales con discapacidad, o instituciones afines, permite el acceso a diversos beneficios.

${ }^{4}$ De acuerdo al MINEDUC, estos programas corresponden a "una estrategia inclusiva del sistema escolar cuyo propósito es entregar apoyos adicionales (en el contexto del aula común) a los estudiantes que presentan Necesidades Educativas Especiales (NEE), sean éstas de carácter permanente o transitorio,” (MINEDUC, 2009).
} 
establecimiento escolar privado con aporte estatal. Sólo un 5\% de los estudiantes egresaron de un establecimiento educacional privado sin aporte estatal.

Respecto al mecanismo de ingreso, un 70\% de los encuestados accedió vía Prueba de Selección Universitaria (PSU), mientras un 32\% ingresó vía Sistema Especial de Admisión, destinada a Discapacitados Visuales. Es necesario señalar que no todos los estudiantes con discapacidad visual acceden por el Sistema Especial, ya que algunos pueden rendir la PSU.

En cuanto a la trayectoria y permanencia en la universidad, un $81 \%$ de los encuestados nunca ha suspendido estudios durante la carrera cursada en la actualidad. Sólo un 19\% ha suspendido estudios. Cabe destacar que los motivos de suspensión no se vincularon a aspectos académicos, y más de la mitad suspendió por motivos de salud física.

Un 87\% de los encuestados no presenta estudios previos de Educación Superior. Solo un 13\% han realizado estudios en otras universidades, de ellos, sólo uno finalizó su carrera. Los otros estudiantes estudiaron su carrera durante 1 o 2 años y 3 de ellos suspendieron sus estudios. Vinculado al aspecto anterior, se puede inferir que estos estudiantes no desertaron del sistema universitario sino que realizaron cambios de institución.

En Chile, las personas financian sus estudios universitarios combinando distintas fuentes, como becas, aportes estatales, créditos bancarios y aportes familiares. En relación a este ítem, los datos señalan que un 55\% de los estudiantes encuestados financia sus estudios con becas, seguido de un $50 \%$ de encuestados que financia sus estudios con fondos estatales, luego un $26 \%$ con créditos bancarios, un $21 \%$ con aporte familiar y un $11 \%$ declara que autofinancia su carrera. Dada la influencia de la condición socio-económica detectada en el FONADIS (2004) es relevante que los estudiantes con discapacidad puedan acceder a becas y ayudas estatales, como sucede en el caso de los encuestados.

\section{Atención educativa a estudiantes con discapacidad}

A) Nivel institucional

La recolección y sistematización de los diversos datos institucionales, posibilitó concluir que la universidad estudiada posee las siguientes atenciones educativas.

1) El Sistema Especial de Admisión para Discapacitados Visuales permite a los estudiantes en esta condición acceder a la Universidad sin rendir la Prueba de Selección Universitaria (PSU), de carácter obligatorio para ingresar a esta universidad. Creado el año 1999, fue el primer decreto que reguló el ingreso vía admisión especial de estudiantes imposibilitados de acceder por el procedimiento estándar.

2) El Programa de Apoyo Multidisciplinario a Estudiantes con Discapacidad Visual, formado el año 1996, tiene por objetivos (a) Apoyar a los alumnos Discapacitados 
Visuales mediante la implementación de una sala de atención en Tiflotecnología, Psicopedagogía y Comunicación. (b) Dar cumplimiento a la Ley 20.422.

3) El Programa Interdisciplinario por la Inclusión, formalizado el año 2013, tiene como objetivo general "apoyar la inclusión de estudiantes con Necesidades Educativas Especiales asociadas a discapacidad en todos los ámbitos de la vida estudiantil, buscando otorgarles equidad e igualdad de oportunidades y condiciones". Su misión es "fomentar la inclusión educativa, apoyando el acceso, permanencia y egreso de estudiantes con discapacidad, así como también desarrollar la formación de profesionales que valoren y respeten la diversidad" (Programa Interdisciplinario por la Inclusión, 2013).

4) La adaptación y construcción de infraestructura destinada a mejorar la accesibilidad de las personas con discapacidad en los tres campus de la universidad. Este trabajo está a cargo de la Dirección de Servicios de la universidad y busca dar cumplimiento a la Ley 20.422.

\begin{tabular}{lcccc}
\hline \multicolumn{1}{c}{ Ítem } & $\begin{array}{c}\text { Campus } \\
\text { Uno }\end{array}$ & $\begin{array}{c}\text { Campus } \\
\text { Dos }\end{array}$ & $\begin{array}{c}\text { Campus } \\
\text { Tres }\end{array}$ & Total \\
\hline Edificios con rampas de acceso & 41 & 9 & 7 & 57 \\
Edificios con rampas de circulación & 5 & 1 & 1 & 7 \\
interior & 18 & 0 & 1 & 19 \\
Edificios con ascensor & 22 & 6 & 4 & 32 \\
Baños para personas con discapacidad & 51 & 2 & 1 & 54 \\
Rampas de conectividad & 2 & 1 & 1 & 4 \\
Dispositivos orugas & & & & \\
\hline
\end{tabular}

Tabla 2: Resumen de infraestructura adaptada en la universidad

Fuente: Elaboración propia basada en datos de Dirección de Servicios

B) Nivel de Servicios

Esta sección tuvo como propósito determinar la satisfacción de los encuestados, respecto a los servicios que entrega la universidad, que no son de exclusivo uso para personas con discapacidad, pero que son utilizados frecuentemente por ellos.

La Dirección de Servicios Estudiantiles, responsable de los servicios complementarios a la actividad académica, también es muy valorada, lo que se demuestra en el alto nivel de satisfacción de los estudiantes encuestados. En particular, el Servicio de Bienestar concentra a un $82 \%$ de estudiantes que se declara satisfecho y muy satisfecho. El Servicio de Salud presenta un $74 \%$ se declaran satisfecho y muy satisfecho (Gráfico 2). No obstante, un $13 \%$ es incluido en la categoría No sabe/ No responde, lo que permite suponer que ellos no utilizan el servicio con una frecuencia que les permita opinar del mismo.

En relación al financiamiento de becas o créditos, un $79 \%$ se declara satisfecho y muy satisfecho, mientras que un $19 \%$ se declara insatisfecho y muy insatisfecho. Esto revela un alto nivel de satisfacción, lo que se puede vincular al hecho que 3 de cada 5 estudiantes poseen una beca. En cuanto al financiamiento para otros gastos además del arancel, las percepciones se dividen de forma similar: un 37\% se declara insatisfecho y 
muy insatisfecho, mientras que un $40 \%$ se declara satisfecho y muy satisfecho (Gráfico 2).

En cuanto al acceso a la información de la universidad ${ }^{5}$, un $61 \%$ se manifiesta satisfecho o muy satisfecho, mientras que un $40 \%$ de los encuestados se encuentra insatisfecho o muy insatisfecho (Gráfico 2). La totalidad de los estudiantes con discapacidad visual manifiestan su insatisfacción con el acceso a la información institucional, debido a la falta de accesibilidad de la plataforma INFODA. Como consecuencia, se impide la apropiada comunicación profesor-estudiante que se realiza a través de este medio. Este es un aspecto crítico que limita considerablemente las condiciones de inclusión que ofrece la universidad a estudiantes con discapacidad visual.

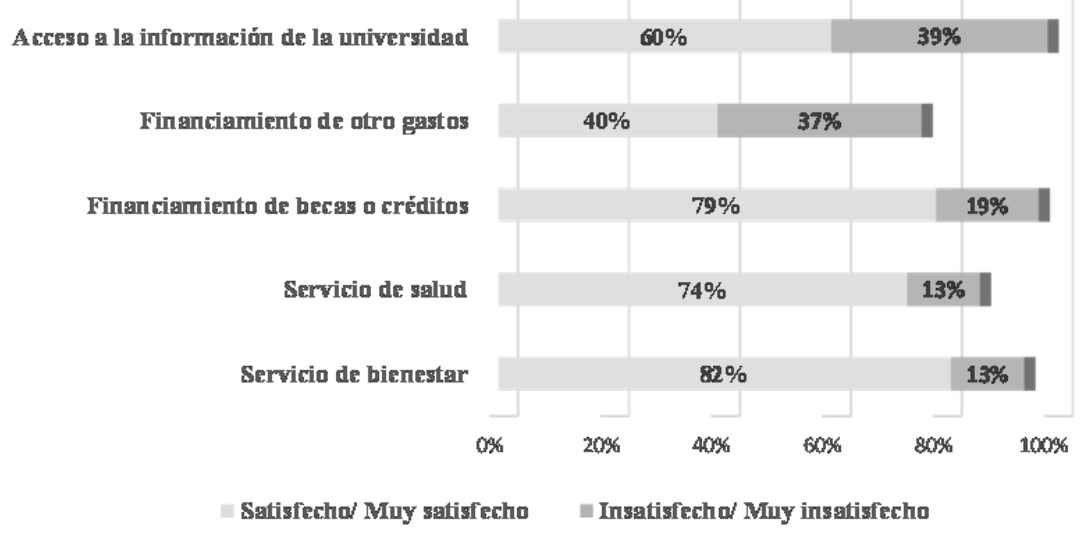

Gráfico 2: Satisfacción con servicios de la universidad

Fuente: Encuesta a estudiantes con discapacidad de la institución, año académico 2013.

\section{C) Nivel de carreras}

Además de los mecanismos facilitadores y servicios de la institución, se consideró identificar la atención educativa brindada desde las carreras cursadas por los estudiantes con discapacidad. Para ello se determinaron tres tipos de apoyo: proceso enseñanza aprendizaje, adecuación de infraestructura y sensibilización.

Del total de estudiantes encuestados, un $68 \%$ sostiene que fue apoyado por su carrera, mientras un 32\% manifiesta lo contrario. Aquellos estudiantes que indicaron haber recibido apoyo, un 54\% declara que se realizaron adaptaciones en los instrumentos de evaluación mientras que un $40 \%$ indica haber recibido apoyo psicopedagógico. La adaptación en los formatos de clase sólo es mencionada por el $16 \%$ de los encuestados. Respecto a la adaptación del espacio físico, las cifras indican 5 Ítem referido a recursos electrónicos institucionales: e-mail, página web institucional y plataforma
INFODA. 
que éste no es frecuente: un 12\% sostiene que se realizó adecuación de mobiliario, mientras que un 16\% declara que se efectuó adecuación de salas y espacios comunes. En cuanto a los aspectos actitudinales de los actores universitarios frente a la discapacidad, se destaca que un 35\% menciona que sus compañeros de estudio estaban sensibilizados ante esta temática. En el caso de los profesores alcanza un 64\% (Gráfico No3).

La encuesta realizada a estudiantes con discapacidad permitió concluir que siete de cada diez encuestados sostiene haber recibido apoyo de su carrera para realizar sus estudios. Entre las principales atenciones educativas se destacan la sensibilización de la comunidad vinculada al encuestado, como son los estudiantes y profesores. Respecto al proceso de enseñanza-aprendizaje, se enfatizó el rol de los profesores en la adaptación de instrumentos de evaluación y el apoyo psicopedagógico.

Corroborando la percepción sobre el rol de los docentes, un 84\% de los encuestados se declara satisfecho y muy satisfecho con la "atención brindada por los profesores", mientras que sólo un $11 \%$ se manifiesta insatisfecho. Este ítem alcanza una de las valoraciones más altas, lo que permite inferir que los profesores son conscientes de la condición de discapacidad de sus estudiantes.

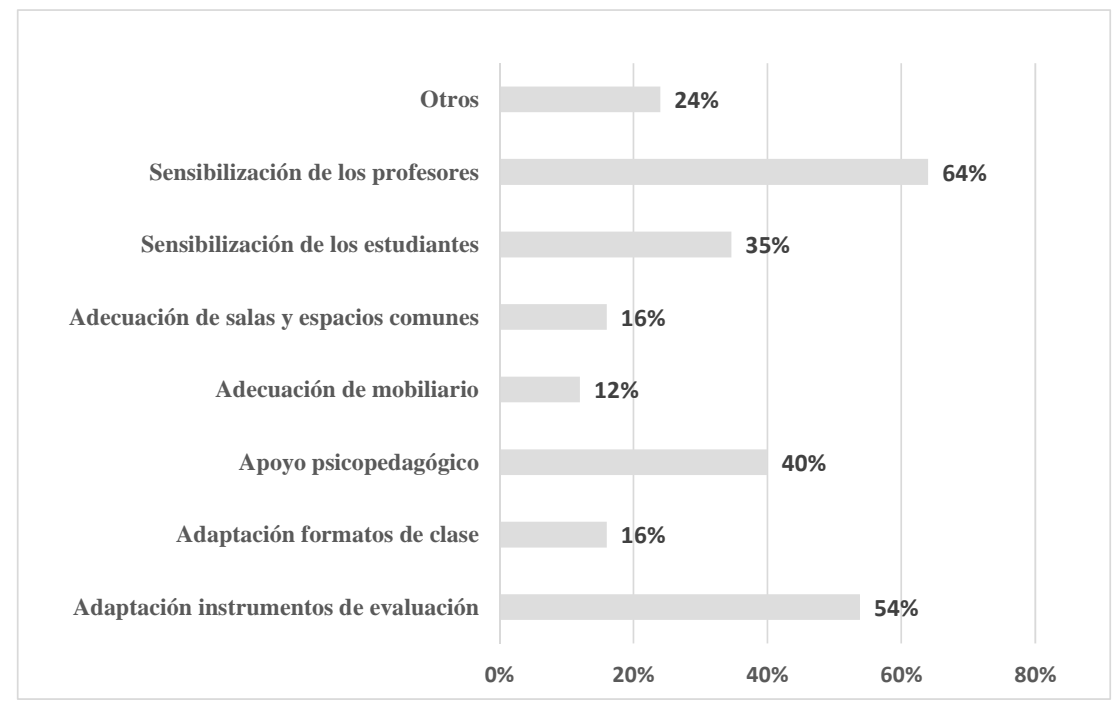

Gráfico 3: Atención educativa brindada por la carrera

Fuente: Encuesta a estudiantes con discapacidad de la institución, año académico 2013.

Percepción de inclusión de los estudiantes con discapacidad en la vida universitaria

La percepción de inclusión fue consultada mediante cuatro ítems referidos a aspectos académicos, integración con los pares, intercambio de vivencias con otros 
estudiantes con discapacidad y experiencias de discriminación en el ámbito universitario.

Se destaca que un $97 \%$ de los encuestados se encuentra de acuerdo y muy de acuerdo con la afirmación: "Me siento capaz de responder a las exigencias académicas de la Universidad". Este dato revela la confianza de los estudiantes en sus propias capacidades y un alta autoestima para superar las dificultades y desafíos de la formación universitaria. Se infiere que estos estudiantes se encuentran empoderados respecto a su formación profesional.

Un 89\% se manifiesta de acuerdo y muy de acuerdo con la afirmación: "Los estudiantes con discapacidad necesitamos de un espacio para intercambiar experiencias”. Este dato es muy relevante porque permite señalar que el programa de inclusión no solo debe ser un mecanismo de gestión de la inclusión a nivel institucional, sino que además una instancia para que los estudiantes compartan sus opiniones y contribuyan mediante sus propias experiencias a la inclusión de otros estudiantes con discapacidad.

Respecto a la frase "Me siento incluido en mi curso como un par", un 87\% está de acuerdo o muy de acuerdo, lo que revela que los estudiantes se perciben como parte de su curso y que la discapacidad no funciona como un impedimento para su inclusión en los espacios formativos de la universidad. No obstante, el $11 \%$ de encuestados se manifiesta contrario a esta afirmación, información que debería ser motivo de preocupación para las autoridades y docentes, puesto que revela percepciones y situaciones negativas en torno a las condiciones de inclusión que entrega la institución.

Un 70\% está en desacuerdo o muy en desacuerdo con la afirmación "me he sentido discriminado/a en la universidad por mi discapacidad". Sin embargo, y vinculado al ítem anterior, un 30\% de los encuestados está de acuerdo o muy de acuerdo con la afirmación. Esto demuestra que aún persisten situaciones de discriminación, que, si bien no son mayoritarias, revelan la necesidad de trabajar en el fortalecimiento de mecanismos de inclusión y de instancias de sensibilización que promuevan la valoración de la diversidad en la vida universitaria.

\section{Discusión y conclusiones}

El acceso y la permanencia en la universidad son elementos fundamentales en la inclusión de personas con discapacidad. En Chile, los antecedentes demuestran que el acceso de estas personas es limitado (FONADIS, 2004). En el estudio realizado se corroboró que la sub-representación de estos estudiantes aún persiste a nivel institucional: 40 estudiantes, es decir, un $0.16 \%$ del total de la institución, muy lejos del 5\% estimado para el grupo entre 15-29 años de la población con discapacidad (FONADIS, 2004). Ahora bien, un dato llamativo se refiere al acceso de las mujeres con discapacidad. Tanto a nivel de sistema de educación superior (CNED, 2014), como los datos recabados por Barros (2011) sobre la matrícula de estudiantes con discapacidad en el sistema, demuestran un acceso bastante igualitario en términos de género. Sin embargo, esta situación no se produce en la institución estudiada, donde 
existe una sub-representación femenina. Por tanto, las mujeres con discapacidad podrían experimentar una doble dificultad en el acceso a esta universidad. Estos resultados requieren de nuevas investigaciones que revelen los orígenes, y, además, que permitan concluir si este dato corresponde a un fenómeno transitorio o permanente, y contribuya a tomar las medidas correspondientes.

La investigación reveló que la permanencia de los estudiantes con discapacidad en la institución es significativa: cuatro de cada cinco nunca ha suspendido estudios durante la carrera cursada. Esto demuestra un alto nivel de retención que, sin embargo, en algunos casos, se combina con un período de permanencia en la carrera más prolongado que lo estimado. En efecto, uno de cada cinco ha permanecido entre siete y nueve años en la universidad. Es necesario profundizar en este fenómeno para determinar la existencia de barreras de aprendizaje que impiden un mejor ajuste al tiempo ideal de duración de las carreras, y a partir de estos antecedentes, generar estrategias específicas. Este punto es especialmente relevante, puesto que a nivel nacional los estudiantes con discapacidad presentan una alta deserción del sistema de educación superior (FONADIS, 2004). Igualmente, es necesario profundizar en los datos de retención y deserción, puesto que en la institución puede existir un fenómeno de deserción "encubierta" de personas con discapacidad, en la medida que no existen registros que indiquen esta condición en aquellos que abandonan sus estudios.

A pesar de que las iniciativas generadas por la institución investigada denotan una preocupación por la inclusión de personas con discapacidad, los resultados permiten concluir que no existe un sistema de gestión que comprenda todas estas iniciativas. En efecto, estas aparecen desarticuladas y sin un referente común que vincule metas, objetivos e indicadores, es decir, no existe una planificación estratégica. Los datos institucionales demuestran que no se han generado protocolos de acción para establecer estándares y procedimiento respecto a los servicios brindados por la universidad.

La universidad no ha establecido protocolos para los profesores que tienen a su cargo el proceso formativo de los estudiantes con discapacidad. A pesar de lo cual ellos reconocen que sus profesores están sensibilizados con su condición. Una manera de materializar esta preocupación es adaptar los instrumentos de evaluación, acción indicada como una de las atenciones educativas más frecuentes. No obstante, esta acción se produce muchas veces sin los conocimientos ni el respaldo oficial necesario, puesto que a nivel institucional no existen programas de capacitación que apoyen a los docentes en el trabajo con personas con discapacidad. La falta de preparación de los profesores ya ha sido identificada en otras universidades por Salinas y otros (2013).

Para avanzar en el mejoramiento de las iniciativas existentes, es fundamental crear un sistema de registro de los estudiantes con discapacidad durante el proceso de matrícula, que permita informar oportunamente a autoridades, profesores y funcionarios la condición del estudiante(s). Además, este sistema se podría ampliar hacia la formalización de protocolos de acción que permitan estandarizar procedimientos, dejando menos espacio a la voluntad de los trabajadores de la universidad, quienes no necesariamente conocen los requerimientos de los estudiantes con discapacidad. En resumen, un sistema de gestión para la inclusión de personas con 
discapacidad debería contribuir a vincular las iniciativas ya existentes, otorgando objetivos, estrategias y acciones compartidas por toda la comunidad universitaria.

El estudio realizado revela que la percepción de los estudiantes acerca de su inclusión en la vida universitaria alcanza una valoración muy positiva, tanto respecto a capacidades académicas para estudiar una carrera universitaria, como respecto a la relación con sus compañeros de generación, y el rol cumplido por sus profesores, quienes son considerados actores clave del apoyo brindado por las respectivas carreras, resultados similares a las conclusiones de Tapia y Monosalva (2012) y Ocampo (2012). A pesar de la positiva percepción de los estudiantes con discapacidad, aún persisten situaciones de discriminación, de acuerdo a lo manifestado por uno de cada cuatro estudiantes. Este dato es una señal que manifiesta la necesidad de generar instancias de sensibilización para toda la comunidad universitaria, potenciando la diversidad como un factor enriquecedor de la formación como persona en la universidad.

Las oportunidades de inclusión a personas con discapacidad en el sistema de educación superior chileno se han desarrollado lentamente durante los últimos años. En este escenario, la institución ha sido pionera en la incorporación a sus aulas de personas con discapacidad. En atención a estos antecedentes, los estudiantes encuestados presentan un alto nivel de expectativas respecto de la atención educativa que brindará la institución para su inclusión. En consecuencia, de acuerdo a sus percepciones, las condiciones de inclusión que ofrece la institución a sus estudiantes con discapacidad se encuentran desarrolladas a un nivel intermedio. En otras palabras, ellos manifiestan un reconocimiento de los esfuerzos institucionales y de la comunidad universitaria, pero, al mismo tiempo, expresan insatisfacción en aspectos importantes. El fortalecimiento de las iniciativas existentes, a través de medidas mencionadas, requiere proyectarse al establecimiento de un sistema de gestión de políticas inclusivas, que se distinga por su carácter sistémico, permitiendo, de este modo, mejores condiciones de inclusión a los estudiantes con discapacidad.

\section{Referencias bibliográficas}

AINSCOW, M., MILES, S. (2008). Por una educación para todos que sea inclusiva: ¿Hacia dónde vamos ahora? Perspectivas Dossier 145 Educación Inclusiva. Unesco y Oficina Internacional de Educación.

BARROS, B. (2011). Estudio de educación superior inclusiva. Ponencia presentada en el Seminario Discapacidad y Educación Superior. Santiago: Universidad Diego Portales.

CASANOVA, M. A. (2011). Educación inclusiva: un modelo de futuro. Editorial Wolters Kluwer, Madrid.

COMISIÓN DE EXPERTOS DE EDUCACIÓN ESPECIAL. (2004). Nueva Perspectiva y Visión de la Educación Especial. Informe de la Comisión de Expertos. Santiago: Ministerio de Educación. 
CONFERENCIA MUNDIAL SOBRE NECESIDADES EDUCATIVAS ESPECIALES. (1994). Acceso y Calidad. Salamanca.

ECHEITA, G., SIMÓN, C., LÓPEZ, M., URBINA, C. (2013). Educación inclusiva, sistemas de referencia, coordinadas y vórtices de un proceso dilemático. En: Verdugo, M.A., Schalock, R. (Coords.). Discapacidad e inclusión. Manual para la docencia. Amarú Ediciones, Salamanca.

ECHEITA, G. (2013). Inclusión y exclusión educativa. De nuevo "voz y quebranto". Revista iberoamericana sobre calidad, eficacia y cambio en educación. Vol. 11, N 2, pp. 99-118.

ECHEITA, G. y AINSCOW, M. (2010). Un marco de referencia y pautas de acción para el desarrollo de sistemas de educación incluyentes. Ponencia presentada por los autores en el II Congreso Iberoamericano de Síndrome de Down. Granada.

ESCUDERO, J., MARTÍNEZ, B. (2011). Educación inclusiva y cambio escolar. Revista Iberoamericana de Educación. N 55, pp. 85-105.

ESTADO DE CHILE. (2010). Ley sobre Igualdad de Oportunidades e Inclusión Social de Personas con Discapacidad. Visitada el 10-12-2013. Disponible en: http://www.senadis.gob.cl/pag/177/557/ley_n20422

FONADIS (2004). Primer estudio nacional de la discapacidad. ENDISC Chile. Apartado Educación Superior. Santiago: INE.

HERNANDEZ, R., FERNANDEZ, C., BAPTISTA, P. (1991). Metodología de la Investigación. Mc México: McGraw-Hill.

LÓPEZ, M. (2011). Barreras que impiden la escuela inclusiva y algunas estrategias para construir una escuela Sin exclusiones. Innovación educativa. № 21, pp. 37-54 37.

MILES, S., SINGAL, N. (2010). The Education for All and inclusive education debate: conflict, contradiction or opportunity? International Journal of Inclusive Education. $\mathrm{N}^{\circ}$. 14:1, pp. 1-15.

MINISTERIO DE EDUCACIÓN (2009). Programa de Integración Escolar (PIE). Decreto Supremo No 170 de 2009. Manual de Orientaciones y Apoyo a la Gestión (Directores y Sostenedores). Santiago: MINEDUC.

MINISTERIO DE EDUCACIÓN (2005). Política Nacional de Educación Especial. Nuestro Compromiso con la Diversidad. Ministerio de Educación. División de Educación General. Santiago: Unidad de Educación Especial.

MINISTERIO DE EDUCACIÓN (2004). Antecedentes históricos, presente y futuro de la educación especial en Chile. Santiago: Programa de Educación Especial.

MORENO, M. T. (2005). Integración/inclusión de las personas con discapacidad en la Educación Superior. En: Informe sobre la educación superior en América Latina y el Caribe 2000-2005: La metamorfosis de la educación superior. Instituto 
Internacional de la UNESCO para la Educación Superior en América Latina y el Caribe. Caracas: IESALC.

OCAMPO, A. (2012). Inclusión de Estudiantes en Situación de Discapacidad a la Educación Superior. Desafíos y oportunidades. Revista Latinoamericana de Educación Inclusiva. 6 (2), 227-239.

ORGANISATION DES NATIONS UNIES POUR L'ÉDUCATION, LA SCIENCE ET LA CULTURE. (2009). Principes directeurs pour l'inclusion dans l'éducation. Paris.

ORGANIZACIÓN DE NACIONES UNIDAS (2006). Convención sobre derechos de personas con discapacidad. Visitada el 18/12/2013 Disponible en: http://www.un.org/esa/socdev/enable/documents/tccconvs.pdf

ORGANIZACIÓN DE LAS NACIONES UNIDAS PARA LA EDUCACIÓN, LA CIENCIA Y LA CULTURA Conferencia Mundial sobre Educación. Visitada el 18/12/2013. Disponible en:

http://www.ibe.unesco.org/fileadmin/user_upload/Policy_Dialogue/48th_ICE/General_ Presentation-48CIE-4__Spanish_.pdf

PROGRAMA INTERDISCIPLINARIO POR LA INCLUSIÓN UDEC (2013). Plan de Trabajo 2013-2014. Documento no publicado.

SALAZAR, C., VALDÉS, F., SANHUEZA, H. y ROSS, M. (2013) Análisis de las buenas prácticas corporativas de la UACh en el proceso de integración universitaria de estudiantes con discapacidad. Revista de Educación Inclusiva, junio, Vol. 6 (2), 59-71.

SALINAS, M., LISSI, M., MEDRANO, D., ZUZULICH, M. y HOJAS, A. (2013). La inclusión en la educación superior: desde la voz de estudiantes chilenos con discapacidad. Revista ibero-americana de educação, 63, 77-98.

TAPIA, C. y MANOSALVA, S. (2012). Inclusión de estudiantes con discapacidad en educación superior. REXE. Revista de Estudios y Experiencias en Educación, 11 (22), 13-34. Universidad Católica de la Santísima Concepción.

VIEYTES, V. (2004). Metodología de investigación en organizaciones, mercado y sociedad: epistemología y técnicas. Buenos Aires: Editorial de las Ciencias.

CONSEJO NACIONAL DE EDUCACIÓN (CNED). (2014). Estadísticas oficiales.

[Base de datos]. Consultado el 08 de marzo de 2014. Disponible en:

http://www.cned.cl/public/Secciones/SeccionIndicesEstadisticas/indices_estadistica s_sistema.aspx

SERVICIO NACIONAL DE DISCAPACIDAD. (2013). Educación Superior. [Texto] Consultado 15 de enero de $2014 . \quad$ Disponible en:http://www.senadis.gob.cl/pag/118/571/educacion_superior 


\section{Correspondencia con los autores}

Gabriela Paulina VILLAFAÑE HORMAZÁBAL

Facultad de Educación,

Universidad de Concepción.

Concepción. Chile.

e-mail: gvillafane@udec.cl

Angélica Aurora CORRALES HUENUL

Facultad de Educación,

Universidad de Concepción.

Concepción. Chile.

e-mail: acorrales@udec.cl

Valentina Javiera SOTO HERNÁNDEZ

Universidad de Concepción.

Concepción. Chile.

e-mail: sotohernandez.valentina@gmail.com 